This is a self-archived version of an original article. This version may differ from the original in pagination and typographic details.

Author(s): Muhonen, Juha T.; La Gala, Giada R.; Leijssen, Rick; Verhagen, Ewold

Title: State Preparation and Tomography of a Nanomechanical Resonator with Fast Light Pulses

Year: 2019

Version: Published version

Copyright: @ 2019 American Physical Society

Rights: In Copyright

Rights url: http://rightsstatements.org/page/InC/1.0/?language=en

Please cite the original version:

Muhonen, J. T., La Gala, G. R., Leijssen, R., \& Verhagen, E. (2019). State Preparation and Tomography of a Nanomechanical Resonator with Fast Light Pulses. Physical Review Letters, 123(11), Article 113601. https://doi.org/10.1103/PhysRevLett.123.113601 


\title{
State Preparation and Tomography of a Nanomechanical Resonator with Fast Light Pulses
}

\author{
Juha T. Muhonen, ${ }^{1,2, *}$ Giada R. La Gala, ${ }^{1}$ Rick Leijssen, ${ }^{1}$ and Ewold Verhagen ${ }^{1, \dagger}$ \\ ${ }^{1}$ Center for Nanophotonics, AMOLF, Science Park 104, 1098 XG Amsterdam, Netherlands \\ ${ }^{2}$ Department of Physics and Nanoscience Center, University of Jyväskylä, P.O. Box 35, FI-40014 University of Jyväskylä, Finland
}

(Received 23 December 2018; published 9 September 2019)

\begin{abstract}
Pulsed optomechanical measurements enable squeezing, nonclassical state creation, and backaction-free sensing. We demonstrate pulsed measurement of a cryogenic nanomechanical resonator with record precision close to the quantum regime. We use these to prepare thermally squeezed and purified conditional mechanical states, and to perform full state tomography. These demonstrations exploit large vacuum optomechanical coupling in a nanophotonic cavity to reach a single-pulse imprecision of 9 times the mechanical zero-point amplitude $x_{\mathrm{zpf}}$. We study the effect of other mechanical modes that limit the conditional state width to $58 x_{\mathrm{zpf}}$, and show how decoherence causes the state to grow in time.
\end{abstract}

DOI: 10.1103/PhysRevLett.123.113601

Measurement and control of mechanical motion at the quantum level is of wide interest because of the quantum technologies it would enable and because of the possibility to probe decoherence in massive systems. Cavity optomechanical demonstrations [1] of quantum control over mechanical resonators have included ground state cooling [2-4], quantum squeezing [5-7], entanglement [8-10], and coupling between mechanical oscillators and qubits $[2,11]$.

Measurement and control are intimately linked. At a basic level, any measurement process is a competition between information gain (measurement rate), decoherence processes, and noise added to the system by the measurement (backaction). If the measurement rate can overcome those detrimental effects, control over the system can be achieved, allowing for example feedback cooling to the ground state [12-14] and the preparation of pure quantum states, conditioned on measurement results $[15,16]$. A continuous measurement of mechanical resonator displacement is subject to the standard quantum limit (SQL). The SQL is a manifestation of Heisenberg's uncertainty principle as the measurement simultaneously observes the two noncommuting motional quadratures, giving a lower limit, equal to the zero-point fluctuation amplitude $x_{\mathrm{zpf}}$, to the noise added by the measurement [17,18]. Evading this backaction limit by moving away from continuous displacement measurements was demonstrated in various quantum systems [19-24], including sideband-resolved opto- or electromechanical cavities probed by two-tone fields [25-27], where the measurement probes only one motional quadrature.

Another method for backaction-evading measurements that has been suggested is that of pulsed measurements $[28,29]$. A single "snapshot" measurement of a harmonic oscillator's position measures one quadrature with potentially unlimited precision, as all backaction is introduced to the orthogonal quadrature with no effect to the future evolution of the measured quadrature. With sufficient precision the mechanical oscillator is then prepared to a squeezed state, conditioned on the measurement. Importantly, the ability to probe all quadratures precisely allows full quantum state tomography [29,30]. Combined with nonlinear measurement or non-Gaussian states of light, pulsed interactions can also induce other nonclassical states [31,32]. Moreover, proposals suggested exploiting pulsed measurement for swap operations between mechanics and light [33] and creation of macroscopic superpositions [32].

Despite these extensive theoretical proposals, so far only a single proof-of-principle experimental demonstration of pulsed optomechanical measurements has been reported [34], at elevated temperature and without cavity enhancement. To reach quantum-level accuracy with a single pulsed measurement one needs to fulfill the challenging requirement $8 \eta \sqrt{N_{P}} g_{0} / \kappa \gtrsim 1$, where $g_{0}$ is the cavity frequency shift for a displacement $x_{\text {zpf }}$ (the vacuum optomechanical coupling rate), $\eta$ the coupling efficiency of light to the cavity, and $N_{P}$ the number of photons in the pulse [29]. At the same time the cavity linewidth $\kappa$ should far exceed the mechanical angular frequency $\omega_{m}$ to accommodate pulse durations much shorter than the mechanical period [35].

In this work, we address these challenges using a cavity optomechanical system based on a sliced photonic crystal nanobeam, allowing large optomechanical coupling rates $[36,37]$. We demonstrate pulsed optomechanical measurements close to the quantum regime, achieving a record-low shot-noise limited single-pulse measurement imprecision of $9 x_{\mathrm{zpf}}$, constrained by optical detection efficiency. We prepare both thermally squeezed and purified (cooled) conditional mechanical states, and perform full state tomography on these. We study how additional mechanical modes affect the conditional state, limiting its width to 


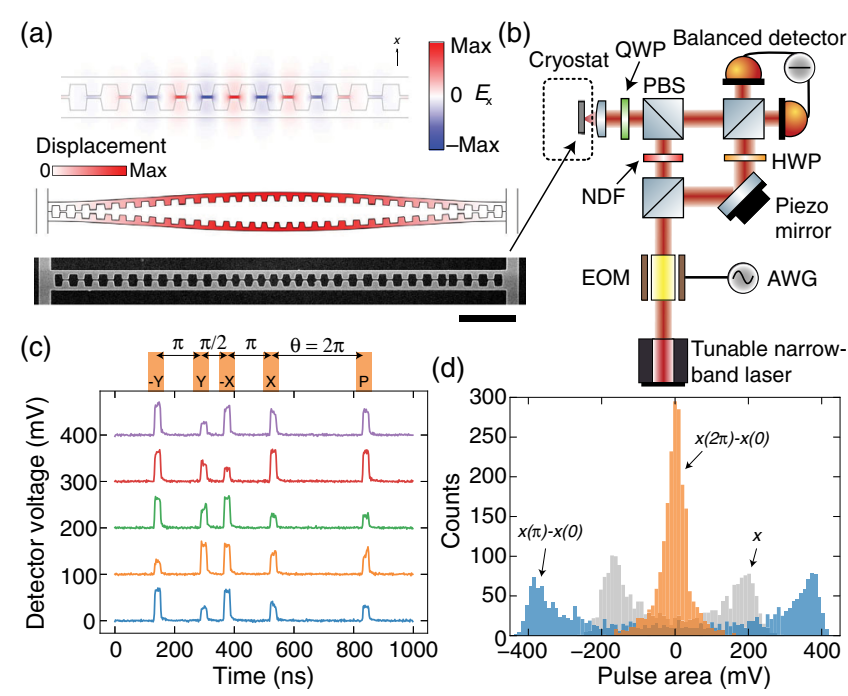

FIG. 1. (a) (bottom) Electron micrograph of the free-standing sliced silicon nanobeam (scale bar $2 \mu \mathrm{m}$ ). (Middle) Simulated displacement of flexural vibrations of the two beam halves. (Top) Simulated transverse electric field of the optical cavity mode. (b) Schematic diagram of the balanced homodyne interferometer setup. Pulses are created by an electro-optical modulator (EOM) controlled by an arbritrary waveform generator (AWG). Quarterwave-plate (QWP), half-wave-plate (HWP), neutral density filter (NDF), and the polarizing beam splitter (PBS) are shown. (c) Example measurement traces, and schematic of the pulsing sequence (separations $\omega_{m} t$ indicated). Traces are offset for clarity. (d) Extracted histograms: thermal state [random sampling, mean subtracted] (gray), difference of two pulses a half-period apart (blue), and difference of two pulses a full period apart (orange).

$58 x_{\mathrm{zpf}}$. We demonstrate how mechanical decoherence and rethermalization can be tracked by recording the state evolution at longer timescales. In addition, we show how postselection allows maximizing sensitivity even though the large optomechanical interaction strength pushes our system deep into the regime of nonlinear optomechanics where sensitivity is reduced [37,38].

Figure 1(a) shows a diagram of the sliced silicon nanobeam. The device, presented in Ref. [37], hosts a photonic crystal nanocavity whose resonance frequency $(204 \mathrm{THz})$ depends very sensitively on the flexural movement of the two beam halves. These move roughly independent of each other with two mechanical mode frequencies around $\omega_{m} /(2 \pi) \approx 3 \mathrm{MHz}$, separated by $\sim 120 \mathrm{kHz}$, and $g_{0} /(2 \pi) \approx 25 \mathrm{MHz}$ approximately equal for both. The optical cavity linewidth $\kappa /(2 \pi) \approx 20.4 \mathrm{GHz}$ enables practically instantaneous measurements of mechanical position while still achieving $g_{0} / \kappa>10^{-3}$. All measurements are performed on the same sample at a temperature of $3.2 \mathrm{~K}$. The exact mechanical frequencies and damping rates drift with time for reasons not fully understood [39].

The sample is incorporated in a homodyne interferometer [Fig. 1(b)]. An electro-optic amplitude modulator produces optical pulses using light from a continuous-wave tunable narrow-band diode laser, which are sent into both interferometer arms. Light is focused on the sample through an $\mathrm{NA} \approx 0.55$ lens, coupling to the nanocavity with efficiency $\eta=\sqrt{\eta_{\text {in }} \eta_{\text {out }}} \approx 0.01$ [37]. Here $\eta_{\text {in,out }}$ are the efficiencies with which light is coupled from the incident laser beam to the cavity and from the cavity to the detectors, respectively. Each incident pulse carries $\sim 2 \times 10^{6}$ photons in a duration $\tau_{P}=20 \mathrm{~ns}$, such that the estimate maximum number of simultaneous intracavity photons is $\sim 60$. The same lens collects emitted cavity radiation, whose phase quadrature is measured by recording the output of a balanced detector after interference with the local oscillator pulse. The resultant detector voltage thus reflects mechanical displacement $x$.

Figure 1(c) shows example recorded pulse traces. Between traces we wait for $30 \mathrm{~ms}$, longer than the mechanical damping time $2 \pi / \Gamma$. One can directly recognize that the recorded pulse heights are correlated when they are separated by a full oscillation period (two last pulses) whereas the pulses separated by half a period (e.g., the third and fourth pulse) are anticorrelated (around a nonzero offset voltage). Figure 1(d) depicts histograms of the difference of the recorded pulses (integrating the voltage over the pulse duration), demonstrating this correlation and anticorrelation behavior. This directly indicates that the thermal nanobeam motion is imprinted on the detected pulses.

To understand the histogram shapes, we need to consider the nonlinearity of the transduction between the mechanical position and optical phase. At the phase-sensitive operation point of the homodyne interferometer and on cavity resonance the balanced detector output is [39]

$$
H=4\left|a_{\text {in }}\right|\left|a_{\mathrm{lo}}\right| \eta \frac{\beta x_{n}}{\beta^{2} x_{n}^{2}+1},
$$

with $\beta=2 g_{0} / \kappa, x_{n}=x / x_{\mathrm{zpf}}$ the normalized displacement and $a_{\text {in }}$ and $a_{\text {lo }}$ the optical field amplitudes towards the sample and in the local oscillator, respectively. This homodyne signal [Fig. 2(a)] depends linearly on displacement only when $\beta x_{n} \ll 1$. Outside this regime, the relationship between signal and displacement is nonlinear and even multivalued. Therefore, our value of $\beta \approx 2.5 \times 10^{-3}$ causes the thermal Gaussian displacement due to both modes with width (square-root of variance) $\sigma_{\text {th }} \approx 290 x_{\mathrm{zpf}}$ to be distorted into a double-peaked probability histogram [Fig. 1(d)]. To calibrate the homodyne signal we fit this thermal histogram to an analytical model using the known sample temperature [39]. The fit allows converting the measurement signal to normalized homodyne signal $H_{\text {norm }}=H / 4\left|a_{\mathrm{s}}\right|\left|a_{\mathrm{lo}}\right| \eta$.

The pulsing sequence in Fig. 1(c) is used for conditional state preparation and tomography. The pulses are separated by an angle $\theta \equiv \omega_{m} t$, where we are considering only one of the mechanical modes. (We address the impact of multiple modes below.) The mechanical resonator motion can be written as $x(\theta)=X \cos (\theta)+Y \sin (\theta)$, using quadrature 

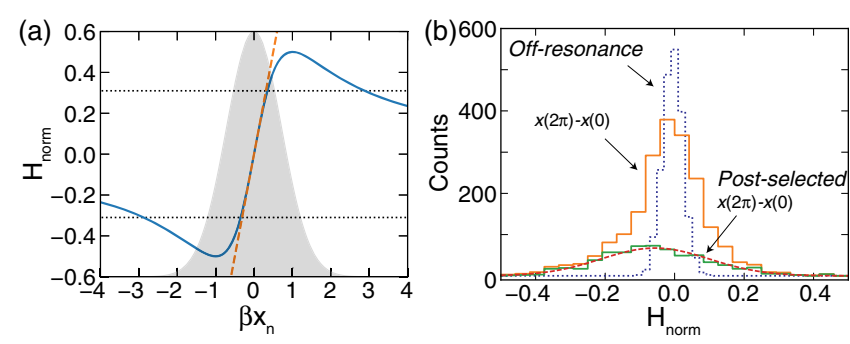

FIG. 2. (a) Full transduction function [Eq. (1), solid line], linear approximation (dashed line), and postselection threshold (dotted lines). Gray area depicts a Gaussian with variance $\beta^{2} \sigma_{\text {th }}^{2}$. (b) The histogram of the difference of two pulses a full period apart [as in Fig. 1(d)], before and after postselection (solid lines). After postselection we recover a Gaussian shape (dashed line shows fit). Dotted line is a reference measurement away from optical resonance, from which the measurement noise floor is extracted.

amplitudes $X, Y$, which vary slowly within pulse trains but are randomized between pulse trains with zero mean and variance $\operatorname{Var}(X)=\operatorname{Var}(Y)=2 n_{\mathrm{th}} x_{\mathrm{zpf}}^{2}$, where $n_{\mathrm{th}}=k_{B} T /\left(\hbar \omega_{m}\right)$ is the number of phonons in thermal equilibrium. The first four pulses of the sequence measure the instantaneous value of the two quadrature amplitudes (state preparation) and the last pulse quantifies the difference between the expected and actual mechanical position (tomography). By varying the waiting time between state preparation and tomography, we can map this difference in all quadrature angles and perform full tomography of the conditional state. We measure both $-X$ and $X$ to cancel measurement offsets caused by low-frequency drifts [39].

To maximize sensitivity and allow single-valued estimation, we postselect the data so that the measured value for the quadrature of interest falls in the linear transduction regime [dotted lines in Fig. 2(a) show the chosen threshold]. Figure 2(b) shows the effect of postselection on the histogram of the difference of two pulses separated by $\theta=2 \pi$. The original histogram had non-Gaussian shape with variance dominated by photon shot noise due to large contributions from parts of the transduction function with reduced sensitivity at $\left|\beta x_{n}\right| \approx 1$. The postselected histogram has a Gaussian shape with larger variance. Hence, the postselection protocol allowed recovering the linear operating regime.

To understand the principles of state preparation, consider an ideal classical measurement at $t=0$, measuring $X$. With just this measurement, the best possible prediction for the oscillator's position at later times is $\cos (\theta) X$, differing from the actual position by $x(\theta)-\cos (\theta) X=\sin (\theta) Y$ [with variance $\sin ^{2}(\theta) \operatorname{Var}(Y)=2 n_{\mathrm{th}} x_{\mathrm{zpf}}^{2} \sin ^{2}(\theta)$ ]. This vanishes at $\theta=\{\pi, 2 \pi\}$, as knowing one quadrature allows predicting the mechanical position exactly every halfperiod. In a phase space defined by the two quadratures this is a squeezed state. Combining two ideal (classical) measurements (a quarter period apart) would then allow measuring both quadratures and preparing a (conditional) pure state with no classical uncertainty in $x$. This state then decays towards thermal equilibrium with time constant $2 \pi / \Gamma$.

In the above idealized case, the pulsed measurements are assumed to be infinitely accurate and backaction-free. In practice, finite measurement imprecision leads to a Gaussian probability distribution for the measured quadrature amplitude. In addition, unavoidably, any measurement disturbs the other quadrature. These intuitions are formalized with a measurement operator given by Caves and Milburn for a free particle [40] and Vanner et al. for harmonic motion [29]

$$
\hat{M}=\frac{1}{\sqrt[4]{\pi}} \exp \left(i \Omega \hat{\mathcal{X}}-\frac{(\hat{\mathcal{X}}-\mathcal{M})^{2}}{2 / \chi^{2}}\right)
$$

where $\mathcal{M}=x_{n}^{\text {meas }} / \sqrt{2}$ with $x_{n}^{\text {meas }}$ the dimensionless measurement result (normalized by $x_{\text {zpf }}$ ), $\hat{\mathcal{X}}=\left(\hat{b}^{\dagger}+\hat{b}\right) / \sqrt{2}=$ $\hat{x} /\left(\sqrt{2} x_{\text {zpf }}\right)$ is the quadrature operator with $\hat{b}$ the phonon annihilation operator, and $\Omega \propto N_{P}$ is the normalized (mean) change of the unmeasured quadrature due to the mean radiation pressure. The parameter $\chi=$ $8 \sqrt{\eta_{\text {in }} \eta_{\text {out }} N_{P}} g_{0} / \kappa[29,32]$ characterizes the pulsed measurement strength and hence the conditional state variance. As mentioned in the introduction, the challenging aim is to achieve $\chi>1$. Physical insight into $\chi$ is provided by noting that a displacement of size $x_{\mathrm{zpf}}$ could be resolved with unity signal-to-noise ratio when comparing two measurements of strength $\chi=1$.

At the limit where $n_{\mathrm{th}} \gg 1$, performing a measurement (transforming the state with $\rho_{f}=\hat{M} \rho_{i} \hat{M}^{\dagger}$, omitting normalization) transforms one quadrature of an arbitrary initial state $\left(\rho_{i}\right)$ into a Gaussian with width $\sigma_{m}=x_{\text {zpf }} / \chi$ and mean given by the random measurement result (following statistics determined by $\rho_{i}$ ), while adding $\Omega \sqrt{2} x_{\text {zpf }}$ to the other quadrature. That quadrature will also gain uncertainty $\sigma_{\mathrm{ba}}=\sqrt{\eta_{\text {in }} / \eta_{\text {out }}} \chi x_{\mathrm{zpf}}$, hence forcing for a state prepared by two sequential measurements a quarter-period apart $\sqrt{\sigma_{m}\left(\sigma_{m}+\sigma_{\text {ba }}\right)} \geq x_{\text {zpf }}$. In Fig. 2(b) we show a histogram measured away from optical resonance to determine the measurement's noise floor. From this we extract a singlepulse measurement imprecision of $\sigma_{m} \approx 9 x_{\mathrm{zpf}}$ [39], corresponding to $\chi \approx 0.11,3$ orders of magnitude higher than the previous state of the art [34]. This value is consistent with measured sample parameters including $\eta_{\text {in }} \approx 1.3 \%$ and for $\eta_{\text {out }} \approx 0.35 \eta_{\text {in }}$, close to our previous independent estimation [37].

Figure 3 presents experimental results of conditional state preparation and tomography. Figure 3(a) shows $X$ and $Y$ obtained from the state preparation. The non-Gaussian distribution shape, as in Fig. 1(d), is related to the nonlinearity; only the blue instances are postselected to be considered for tomography. Scanning the delay between 


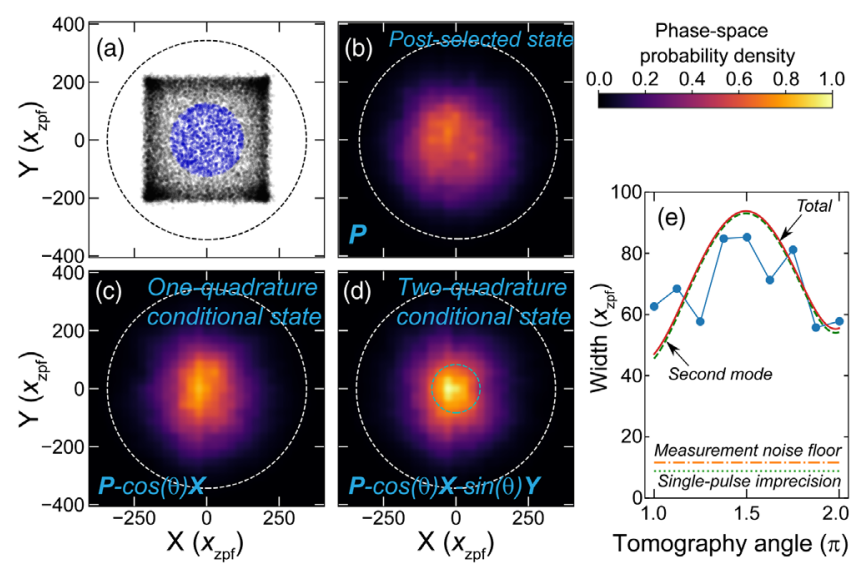

FIG. 3. (a) Values of $X$ and $Y$ from state preparation pulses, post-selected measurements as blue data points. (b)-(d) Reconstructed phase-space probability densities, for unconditional data (b), one-quadrature conditional data (c) and two-quadrature conditional data (d). A single normalization is applied on panels (b)-(d). White dashed lines show the calculated full-width-halfmaximum (FWHM) of the thermal state at $3.2 \mathrm{~K}$, and green dashed line the measured FWHM of the conditional state. Reconstructions use nine different angles $\theta$ each combining 2000 acquired traces. (e) Width of mechanical marginals for the two-quadrature conditional data. Dash-dotted line shows the measurement noise floor, dashed line the expected second mechanical mode contribution, and solid line their squared sum. The noise floor differs slightly from the singlepulse imprecision (dotted line) as the measurement uses multiple pulses and shot noise of the tomography pulse is not subtracted [39].

preparation pulses and the tomography pulse (i.e., varying $\theta$ ) allows mapping the mechanical marginals and reconstructing the phase-space probability density of the conditional state via an inverse Radon transform from the histograms [29,41]. As we subtract the measured (random) values for the quadrature amplitudes, the plots depict the conditional state shifted to origin. Figure 3(b) shows this Radon transform for only the tomography pulse, depicting a small central part of the thermal Gaussian distribution, determined by the postselection. Figure 3(c) shows the thermally squeezed state prepared with single-quadrature conditionalization. The state is extracted with $P-\cos (\theta) X$, with $P$ the result of the tomography pulse and $X$ the measured quadrature amplitude from the previous pulses. Finally, in Fig. 3(d) we plot the phase-space probability density for the state conditionalized in both quadratures $P-\cos (\theta) X-\sin (\theta) Y$, which would have the ground state area if the measurements would be ideal. In our case the state has an average width that corresponds to a one-mode thermal state at a temperature of $380 \mathrm{mK}$, purified from the original temperature of $3.2 \mathrm{~K}$.

The width of this conditional state is plotted in Fig. 3(e) as a function of the tomography angle. In the measured data the minimum width reached is $58 x_{\mathrm{zpf}}$, significantly

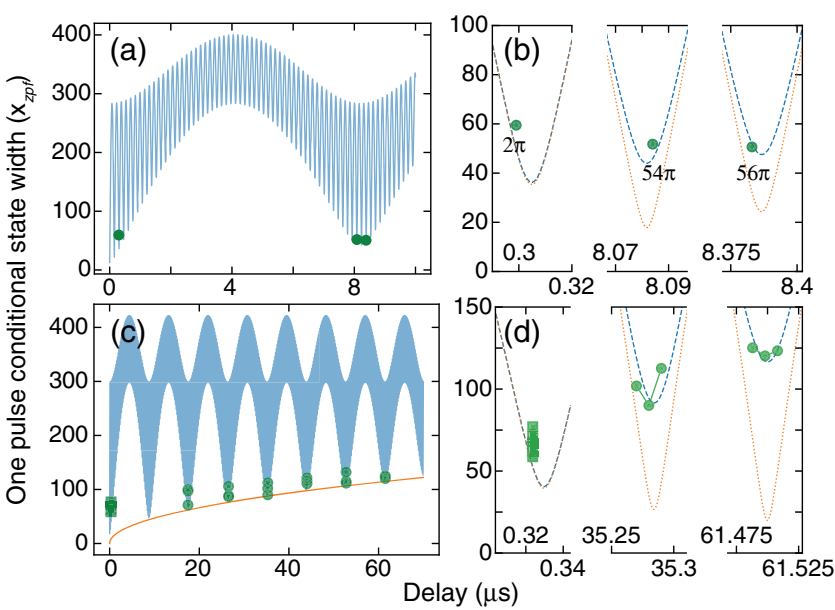

FIG. 4. (a)-(b) Common mode measurement, smaller conditional state width is achieved at $\theta=54 \pi, 56 \pi$ than at $\theta=2 \pi$ due to minimization of the second mode contribution. Panel (b) shows close-ups of (a). Blue line shows expected behavior with fitted $\Gamma /(2 \pi)=400 \mathrm{~Hz}$, yellow dashed line in (b) without any decoherence. Circles are measured data. (c)-(d) Thermal decoherence measurement demonstrating the growing conditional state width. Panel (d) shows close-ups of (c). Yellow line in (c) is the envelope function $\sqrt{[1-\exp (-t \Gamma / 2)] 8 n_{\mathrm{th}}} x_{\mathrm{zpf}}$, and blue line the full expected curve, both with fitted $\Gamma /(2 \pi)=400 \mathrm{~Hz}$, yellow dashed line in (d) without decoherence. Each pulse sequence here has two tomography pulses, one at $\theta=2 \pi$ (shown as squares) and other at variable distance (circles). Also shown is data at $\pm 10 \mathrm{~ns}$ around $\theta=2 n \pi$ points. All datasets contained 1000 samples before postselection.

exceeding the shot noise floor, and is maximized at $\theta=3 \pi / 2$. These features are explained through the existence of a second mechanical mode. As in this device the two mechanical modes couple equally strongly to the cavity, the contribution of the second mechanical mode is captured with $x(\theta)=X_{1} \cos (\theta)+Y_{1} \sin (\theta)+X_{2} \cos (r \theta)+$ $Y_{2} \sin (r \theta)$, with $r$ the ratio of the mechanical frequencies and subscripts refer to the modes. The resulting uncertainty caused by the second mode [39] as a function of $\theta$ is plotted in Fig. 3(e), matching the data well. There are no fitting parameters here, as both modes' frequency and temperature are known. The nonmonotonic shape is caused by measuring the $Y$ quadrature before the $X$ quadrature, causing it to have a larger variance as it has more time to evolve out of sync with the mode of interest [39]. Assuming $r \approx 1$ and equal $n_{\text {th }}$ and $x_{\text {zpf }}$ for both modes, the expected contribution to the conditional state width from the second mode can be approximated as $\sqrt{4 n_{\mathrm{th}}[1-\cos (r \theta) \cos (\theta)-\sin (r \theta) \sin (\theta)]} x_{\mathrm{zpf}}$ [39]. This vanishes when $\cos (\theta) \cos (r \theta)=1$ and $\sin (\theta) \sin (r \theta)=0$, or vice versa. Although this cannot be fulfilled exactly unless $r$ is a rational number, it is approximated when $\theta=2 n \pi$, where $n \approx \omega_{m} /\left(r \omega_{m}-\omega_{m}\right)$. In Figs. 4(a)-4(b) we compare the one-pulse conditional state width after one mechanical period $(\theta=2 \pi)$ and where this condition 
is fulfilled $(n \approx 54,56)$. A slightly lower conditional state width is measured at $\theta=54 \pi, 56 \pi$ than at $\theta=2 \pi$.

The state widths at $\theta=54 \pi, 56 \pi$ differ strongly from expectation based on the formula above. This is because we neglected thermal decoherence, which will cause the minimum conditional state width to increase in time as $\sqrt{8 n_{\mathrm{th}}[1-\exp (-t \Gamma / 2)]} x_{\mathrm{zpf}}$ for two mechanical modes, assuming the modes have identical $n_{\text {th }}, x_{\mathrm{zpf}}$, and $\Gamma$ [39]. Figures 4(c)-4(d) show measurements around the times where the second mode contribution should vanish on a longer timescale, tracking the loss of coherence due to thermalization quantitatively in time domain.

These results show that nano-optomechanical systems can bring quantum-level mechanical measurements with single nanosecond pulses within reach. Notably, achieving $\eta \gtrsim 8 \%$ without changing any other parameters would bring the uncertainty in one quadrature below $x_{\mathrm{zpf}}$, allowing squeezed state preparation and observing quantum backaction [18]. Preparing a pure state of a single resonator would require reducing the second mode contribution, through (opto)mechanical design (coupling the two beam halves more strongly creating a single optically bright mode), or by further cooling (cryogenic or feedback). Alternatively, one could exploit the fact that with quantum-level precision, a single pulse entangles the quadratures of the two mechanical modes, providing a new path to explore many-mode quantum optomechanics in the time domain.

Indeed, our experiments demonstrate how pulsed measurements yield interesting possibilities for measurement and control of mechanical motion, complementing the conventional frequency domain analyses. This "timedomain optomechanics" could allow new protocols for quantum sensing that exploit fast backaction-free determination of a mechanical quadrature, as well as new paradigms to create quantum states of motion and mechanical entanglement.

The authors thank Hugo Doeleman for critical reading of the manuscript. This work is part of the research programme of the Netherlands Organisation for Scientific Research (NWO), and supported by the European Union's Horizon 2020 research and innovation programme under Grant Agreement No. 732894 (FET Proactive HOT). E. V. gratefully acknowledges an NWO-Vidi grant for financial support. J. T. M. thankfully acknowledges funding from the European Union's Horizon 2020 research and innovation programme under the Marie Skłodowska-Curie Grant Agreement No. 707364.

*juha.t.muhonen@jyu.fi

verhagen@amolf.nl

[1] M. Aspelmeyer, T. J. Kippenberg, and F. Marquardt, Rev. Mod. Phys. 86, 1391 (2014).
[2] A. D. O'Connell, M. Hofheinz, M. Ansmann, R. C. Bialczak, M. Lenander, E. Lucero, M. Neeley, D. Sank, H. Wang, M. Weides, J. Wenner, J. M. Martinis, and A. N. Cleland, Nature (London) 464, 697 (2010).

[3] J. Chan, T. P. M. Alegre, A. H. Safavi-Naeini, J. T. Hill, A. Krause, S. Gröblacher, M. Aspelmeyer, and O. Painter, Nature (London) 478, 89 (2011).

[4] J. D. Teufel, T. Donner, D. Li, J. W. Harlow, M. S. Allman, K. Cicak, A. J. Sirois, J. D. Whittaker, K. W. Lehnert, and R. W. Simmonds, Nature (London) 475, 359 (2011).

[5] E. E. Wollman, C. U. Lei, A. J. Weinstein, J. Suh, A. Kronwald, F. Marquardt, A. A. Clerk, and K. C. Schwab, Science 349, 952 (2015).

[6] J.-M. Pirkkalainen, E. Damskägg, M. Brandt, F. Massel, and M. A. Sillanpää, Phys. Rev. Lett. 115, 243601 (2015).

[7] F. Lecocq, J. B. Clark, R. W. Simmonds, J. Aumentado, and J. D. Teufel, Phys. Rev. X 5, 041037 (2015).

[8] K. C. Lee, M. R. Sprague, B. J. Sussman, J. Nunn, N. K. Langford, X.-M. Jin, T. Champion, P. Michelberger, K. F. Reim, D. England, D. Jaksch, and I. A. Walmsley, Science 334, 1253 (2011).

[9] R. Riedinger, A. Wallucks, I. Marinkovi, C. Löschnauer, M. Aspelmeyer, S. Hong, and S. Gröblacher, Nature (London) 556, 473 (2018).

[10] C. F. Ockeloen-Korppi, E. Damskägg, J.-M. Pirkkalainen, M. Asjad, A. A. Clerk, F. Massel, M. J. Woolley, and M. A. Sillanpää, Nature (London) 556, 478 (2018).

[11] Y. Chu, P. Kharel, W. H. Renninger, L. D. Burkhart, L. Frunzio, P. T. Rakich, and R. J. Schoelkopf, Science 358, 199 (2017).

[12] P. F. Cohadon, A. Heidmann, and M. Pinard, Phys. Rev. Lett. 83, 3174 (1999).

[13] D. J. Wilson, V. Sudhir, N. Piro, R. Schilling, A. Ghadimi, and T. J. Kippenberg, Nature (London) 524, 325 (2015).

[14] M. Rossi, D. Mason, J. Chen, Y. Tsaturyan, and A. Schliesser, Nature (London) 563, 53 (2018).

[15] H. M. Wiseman and G. J. Milburn, Quantum Measurement and Control (Cambridge University Press, Cambridge, England, 2009).

[16] M. Rossi, D. Mason, J. Chen, and A. Schliesser, arXiv: 1812.00928.

[17] K. W. Murch, K. L. Moore, S. Gupta, and D. M. StamperKurn, Nat. Phys. 4, 561 (2008).

[18] T. P. Purdy, R. W. Peterson, and C. A. Regal, Science 339, 801 (2013).

[19] G. Vasilakis, H. Shen, K. Jensen, M. Balabas, D. Salart, B. Chen, and E. S. Polzik, Nat. Phys. 11, 389 (2015).

[20] C. F. Ockeloen-Korppi, E. Damskägg, J.-M. Pirkkalainen, A. A. Clerk, M. J. Woolley, and M. A. Sillanpää, Phys. Rev. Lett. 117, 140401 (2016).

[21] C. B. Møller, R. A. Thomas, G. Vasilakis, E. Zeuthen, Y. Tsaturyan, M. Balabas, K. Jensen, A. Schliesser, K. Hammerer, and E.S. Polzik, Nature (London) 547, 191 (2017).

[22] N. S. Kampel, R. W. Peterson, R. Fischer, P.-L. Yu, K. Cicak, R. W. Simmonds, K. W. Lehnert, and C. A. Regal, Phys. Rev. X 7, 021008 (2017).

[23] V. Sudhir, R. Schilling, S. A. Fedorov, H. Schütz, D. J. Wilson, and T. J. Kippenberg, Phys. Rev. X 7, 031055 (2017). 
[24] D. Mason, J. Chen, M. Rossi, Y. Tsaturyan, and A. Schliesser, Nat. Phys. 15, 745 (2019).

[25] J. B. Hertzberg, T. Rocheleau, T. Ndukum, M. Savva, A. A. Clerk, and K.C. Schwab, Nat. Phys. 6, 213 (2010).

[26] J. Suh, A. J. Weinstein, C. U. Lei, E. E. Wollman, S. K. Steinke, P. Meystre, A. A. Clerk, and K. C. Schwab, Science 344, 1262 (2014).

[27] I. Shomroni, L. Qiu, D. Malz, A. Nunnenkamp, and T. J. Kippenberg, Nat. Commun. 10, 2086 (2019).

[28] V. B. Braginsky, Y. I. Vorontsov, and F. Y. Khalili, JETP Lett. 27, 276 (1978).

[29] M. R. Vanner, I. Pikovski, G. D. Cole, M. S. Kim, Č. Brukner, K. Hammerer, G. J. Milburn, and M. Aspelmeyer, Proc. Natl. Acad. Sci. U.S.A. 108, 16182 (2011).

[30] M. R. Vanner, I. Pikovski, and M. S. Kim, Ann. Phys. (Amsterdam) 527, 15 (2015).

[31] M. R. Vanner, Phys. Rev. X 1, 021011 (2011).

[32] U. B. Hoff, J. Kollath-Bönig, J. S. Neergaard-Nielsen, and U. L. Andersen, Phys. Rev. Lett. 117, 143601 (2016).
[33] J. S. Bennett, K. Khosla, L. S. Madsen, M. R. Vanner, H. Rubinsztein-Dunlop, and P. Warwick, New J. Phys. 18, 053030 (2016).

[34] M. R. Vanner, J. Hofer, G. D. Cole, and M. Aspelmeyer, Nat. Commun. 4, 2295 (2013).

[35] As $N_{P}$ is in practice limited due to nonlinear optical effects such as two-photon absorption, this makes large magnitudes of $g_{0}$ beneficial.

[36] R. Leijssen and E. Verhagen, Sci. Rep. 5, 15974 (2015).

[37] R. Leijssen, G. R. La Gala, L. Freisem, J. T. Muhonen, and E. Verhagen, Nat. Commun. 8, 16024 (2017).

[38] G. A. Brawley, M. R. Vanner, P. E. Larsen, S. Schmid, A. Boisen, and W. P. Bowen, Nat. Commun. 7, 10988 (2016).

[39] See Supplemental Material at http://link.aps.org/ supplemental/10.1103/PhysRevLett.123.113601 for further experimental and theoretical details and supplemental experimental results.

[40] C. M. Caves and G. J. Milburn, Phys. Rev. A 36, 5543 (1987).

[41] S. van der Walt, J. L. Schönberger, J. Nunez-Iglesias, F. Boulogne, J. D. Warner, N. Yager, E. Gouillart, T. Yu, and (The Scikit-image contributors), PeerJ 2, e453 (2014). 